\title{
Roberte Hamayon, Jouer. Une étude anthropologique à partir d'exemples sibériens
}

Paris, La Découverte, coll. « Bibliothèque du Mauss », 2012, 369 p.

Nathalie Luca

\section{(2) OpenEdition}

\section{Journals}

Édition électronique

URL : http://journals.openedition.org/assr/25502

DOI : $10.4000 /$ assr.25502

ISSN : $1777-5825$

Éditeur

Éditions de l'EHESS

Édition imprimée

Date de publication : 30 décembre 2013

Pagination : 210

ISSN : 0335-5985

Référence électronique

Nathalie Luca, "Roberte Hamayon, Jouer. Une étude anthropologique à partir d'exemples sibériens », Archives de sciences sociales des religions [En ligne], 164 | 2013, mis en ligne le 03 mars 2014, consulté le 21 septembre 2020. URL : http://journals.openedition.org/assr/25502 ; DOI : https://doi.org/ $10.4000 /$ assr. 25502

Ce document a été généré automatiquement le 21 septembre 2020.

(c) Archives de sciences sociales des religions 


\section{Roberte Hamayon, Jouer. Une étude anthropologique à partir d'exemples sibériens}

Paris, La Découverte, coll. « Bibliothèque du Mauss », 2012, 369 p.

Nathalie Luca

\section{RÉFÉRENCE}

Roberte Hamayon, Jouer. Une étude anthropologique à partir d'exemples sibériens, Paris, La Découverte, coll. « Bibliothèque du Mauss », 2012, 369 p. 
On connaît Roberte Hamayon pour ses remarquables travaux sur le chamanisme, dont elle a écrit la première théorie sous le beau titre de La chasse à l'âme (1990), ouvrage qui traite plus largement, à partir de l'exemple sibérien, de la gestion de l'aléatoire liant "la "chance", en tant que bien symbolique, le "jeu" comme technique d'obtention de ce bien, et la "croyance" comme attitude mentale qui conditionne cette obtention " (Buffetrille, Lambert, Luca, de Sales, Études mongoles et sibérienne, centrasiatiques et tibétaines, 2013, hors série, 14). Ayant de ce fait travaillé de longue date sur la notion de jouer, dont elle remarque qu'elle est étrangement restée le parent pauvre de l'anthropologie, on ne s'étonnera pas qu'elle lui consacre aujourd'hui la première théorie dans ce champ

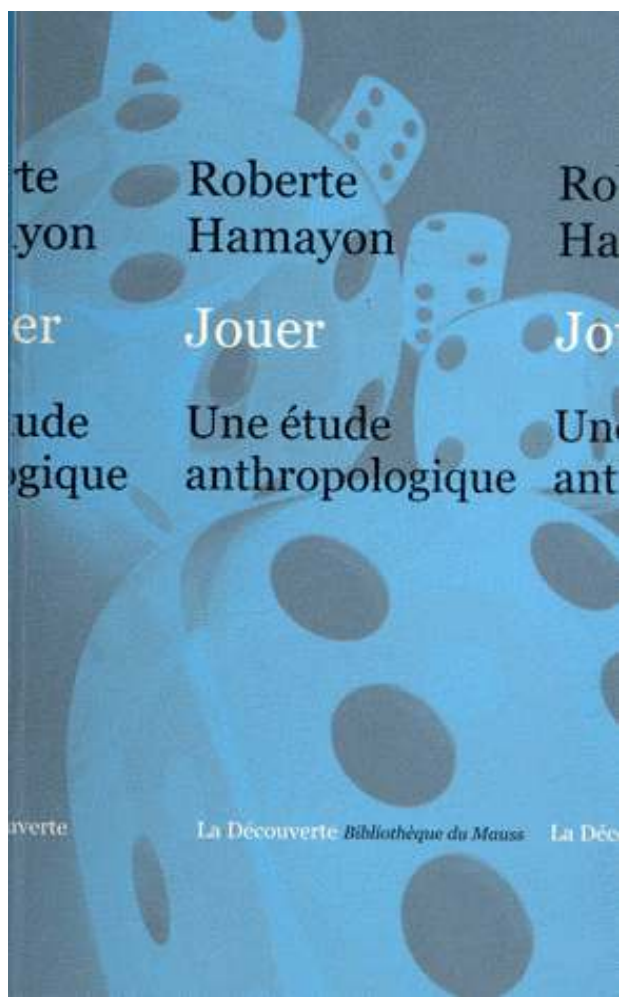
disciplinaire. Sa préférence pour la forme verbale plutôt que nominale le jouer indique que sa réflexion ne s'attache pas à dégager des catégories ou à réaliser une typologie des activités ludiques, mais à mettre au jour un processus, une façon d'agir intrinsèque au jeu. Ce faisant, elle reprend sous un angle différent la combinaison déjà posée entre chance, jeu et croyance. Aucun doute qu'il manque encore un opus sur le croire pour clore ce triptyque...

2 Roberte Hamayon s'attache déjà à expliquer l'intérêt de constituer le jouer en objet de recherche, et plus encore, en concept générique apte à englober des thèmes proches mais jusque-là traités à part comme le sport, le rituel, le combat ou la danse dont elle explique qu'ils ont été détachés de leur matrice originelle en même temps qu'ils ont été chassés de l'église. Dès le départ en effet, l'existence d'une dimension sacrificielle, attachée aux jeux romains, a fait craindre à Tertullien leur caractère trompeur et idolâtre. Par la suite, la condamnation des jeux par l'Église a reposé sur deux arguments: "l'exhibition de mœurs dissolues et le divertissement théâtral, qui soustrait du temps à la prière » (p. 61). Dans les deux cas, la réprobation portait sur la dimension corporelle de ces conduites, en particulier le saut et la danse sautée, diabolisée pour son aptitude à évoquer l'animal. Il fallut cependant attendre la fin du $\mathrm{XVI}^{\mathrm{e}}$ siècle pour que le chrétien parvienne à laisser l'expressivité de son corps à la porte de l'église afin d'y mieux pénétrer en esprit. Le corps fut alors "dressé » au sein de structures laïques de plus en plus spécialisées, où jeu et sport se dédoublèrent au détriment du caractère totalisateur et sacré que possédait le premier à l'origine. Les jeux ont continué d'être utilisés comme technique d'obtention de la chance, laissant au jouer son "rôle préparatoire " tout à fait primordial, c'est-à-dire sa capacité de «mobiliser le sens de la temporalité et d'ouvrir sur l'avenir » (p. 141), de déconnecter l'action faite durant le jeu de ce qui en est attendu dans la vie réelle. Tout au contraire, le sport, par le renforcement de sa dimension compétitive, en est venu à représenter la 
force de l'homme, du groupe ou de la nation, accompagnant «le processus de centralisation hiérarchique au sein de la société [...] la construction du pouvoir politique emportant l'identité collective » (p. 172-174), tout en abolissant la distinction entre l'action préparatoire et l'action préparée : «Le jouer, alors, ne prépare que luimême; il se rationalise sur la base de critères mesurables à la poursuite du perfectionnement perpétuel. [...] C'est l'un des signes du passage du jeu au sport.» (p. 142-144) Tout ce qui n'est pas sanctionnable, tels la danse et autres jeux mixtes reposant sur la complémentarité des rôles, est bientôt rejeté aux marges de la société moderne, dans ses pratiques folkloriques, tandis que la lutte et l'ensemble des jeux masculins de compétitivité et d'opposition incarnent ses valeurs.

3 Tout en posant les conditions historiques du rejet progressif du jeu, Roberte Hamayon essaie d'en extraire la structure active en partant de la notion de cadre fictionnel empruntée à Gregory Bateson. Celui-ci l'a utilisée pour expliciter ce que peut représenter «la morsure ludique» de deux singes s'amusant à se défier dans un zoo. Cela ressemble à une morsure, mais ce n'en est pas vraiment une. Pourtant, à l'intérieur du cadre, il constate qu'elle est considérée comme telle: «J'ai vu jouer deux jeunes singes; autrement dit, deux singes engagés dans une séance interactive dont les unités d'action, ou signaux, étaient analogues, mais non pas identiques à ceux du combat. [...] Il était évident que, pour les singes eux-mêmes, ceci était "un jeu". » (Bateson, Vers une écologie de l'esprit, tome 1, Paris, Le Seuil, «Essais », 1977, p. 249) Bateson en déduit que la capacité même de jouer passe nécessairement par un degré de métacommunication indiquant aux acteurs qu'ils sont dans une séquence de jeu.

4 Le cadre fictionnel, tel qu'il l'a défini, avait déjà inspiré plusieurs anthropologues. Parmi eux se trouve notamment Albert Piette : «Dans le même sens selon lequel il n'y a pas de morsure dans le jeu des animaux, remarque-t-il, Jésus n'est pas réellement présent (au sens dur, factuel du terme) à la messe, comme le reconnaîtraient la plupart des chrétiens et, de la même façon, l'opposition entre les deux villes n'est pas réelle dans le match de football qui les oppose. Dans ces deux exemples, il y a extraction de deux types différents de données, une situation historique qui a eu lieu (la Cène) et l'opposition, en tout cas la différence entre deux cités (deux pays); ensuite recontextualisation de ces données sous un cadre ludique. » Mettre une action dans un cadre fictionnel revient ainsi, pour Piette, à indiquer que « ce qui est dit ou fait ne peut pas être pris littéralement: l'arrivée du Christ n'est pas attendue le dimanche à 11 heures, et il n'y a aucune stratégie géopoliticomilitaire entre les équipes de football ». Pour autant précise-t-il, cela ne veut pas dire non plus que "le Christ est absent » du culte et que les matchs de football se déroulent sans agressivité aucune. Cependant, à l'intérieur du cadre, on ne risque pas les conséquences concrètes qu'auraient ces situations si elles se produisaient vraiment (Piette, "Pour une anthropologie comparée des rituels contemporains », Terrain, 29, 1997, p. 139-150).

5 Reprenant à son tour une lecture de ce cadre fictionnel à partir d'exemples prélevés chez les Bouriates, dans le chamanisme, Roberte Hamayon apporte pour sa part une distinction entre ce qui se passe dans le jeu, qui relève de l'imitation, ce qui se passe dans le rite chamanique, qui relève de la simulation et dans la performance épique, qui «substitue la forme intellectualisée de la narration chantée à celle de la simulation gestuelle»(p.145). Elle admet, comme Piette, que les joueurs imitent certaines attitudes tout en étant exemptés des suites que les actions imitées impliquent dans la vie réelle. Cependant, dans le rite, il en va autrement. Les acteurs simulent dans le 
cadre fictionnel des actions censées se passer vraiment à un autre niveau, celui du monde des invisibles, dans lequel elles ne sont nullement exemptées des suites qui en sont attendues. Elles sont de surcroît supposées avoir des répercussions, d'un autre ordre cette fois, sur la vie ordinaire, réelle de la communauté : «Alors que le lutteur donnait réellement des coups de tête à son rival dans un cadre impliquant qu'il ne combattait pas, le chamane fait l'inverse : il donne des coups de tête en l'air et le cadre rituel impose de comprendre qu'il se bat vraiment, et cela contre un rival invisible dans le monde des invisibles.» (p.127) En définitive, le rite apporte au jeu une structure d'interaction sur deux plans interdépendants: entre humains d'une part, et entre monde humain et monde spirituel d'autre part. C'est ainsi par la plus grande complexité de sa dimension interactive qu'il se caractérise. De là surgissent d'autres compléments. Ainsi, si le jeu comporte une dimension imitative (le joueur imite des gestes qui existent hors du cadre du jeu), une dimension préparatoire (le joueur apprend des gestes qui lui serviront dans la vie ordinaire, ce qui suppose une interférence du jeu avec la vie de tous les jours), une dimension cognitive et interactionnelle (le joueur découvre des rapports d'identité et d'altérité; de complémentarité - pour ce qui est de la danse - et d'opposition - en ce qui concerne la lutte), une dimension d'agentivité (les objets devenant dans le jeu des partenaires vivants, à part entière), le rite $\mathrm{y}$ ajoute une dimension simulative (distinction entre ce que le chamane fait dans le cadre fictionnel du jeu et ce qu'il réalise vraiment dans la surnature), une dimension préparatoire différenciée (distinction entre ce qui est attendu du rite dans la vie ordinaire - de la chance à la chasse, notamment - et ce qu'il accomplit dans la surnature - un mariage), une dimension performative essentielle (puisqu'il est donc attendu du jeu rituel un "effet » sur la réalité) et finalement une dimension cognitive qui relie le jouer au croire (le rite assurant l'apprentissage des signes de la présence de l'invisible). Plus encore, le rite apparaît en dernière instance comme une façon de se jouer des esprits, car il est construit de sorte à donner l'avantage aux humains, et cela vaut peut-être plus encore pour les épopées que pour les jeux rituels chamaniques : " chants épiques et histoires à rire ont beau ne pas faire partie du "jouer", ils se présentent comme des extensions de ce jouer et se situent dans la continuité : ils visent également à donner l'avantage à l'humain qui y a recours, confirmant l'orientation active de l'acte de jouer et le privilège de celui qui en a l'initiative. Que les humains jouent, chantent ou rient, ils "se jouent" des esprits " (p. 186).

6 Dès lors se pose une nouvelle question : celle de la différentiation entre jeu rituel et autres modalités du rituel (prière, sacrifice, etc.). La réponse se trouve précisément dans la centralité de la simulation du premier : «les prières (chantées) et les sacrifices et offrandes (libations et aspersions) rendent les ancêtres directement "présents" en tant que destinataires » (p. 203). Il n'est donc pas besoin de les représenter. Même si les gestes des fidèles laissent percevoir le type de relations qu'ils entretiennent avec eux, personne ne prend en charge leur réaction: elle n'est pas donnée à voir. Tout au contraire, quand le chamane chamanise, il montre qu'il implique l'esprit sur le moment même de l'action. Ainsi faut-il encore souligner la dimension représentative essentielle du jeu rituel.

7 C'est arrivé à ce niveau de définition du jouer qu'une réflexion sur le croire devient incontournable. Roberte Hamayon présente ainsi la question qui se pose: «quelle validité, quelle portée [les joueurs] accordent-ils aux actions en train de se faire que les jeux représentent?» (p. 206) Cela suppose, remarque-t-elle, de faire sciemment le 
choix d'être dupe, d'adhérer sciemment à l'univers fictionnel, et par conséquent, de ressentir sincèrement les émotions qu'appellent les actions qui y sont accomplies. Ainsi jouer, c'est avant tout commettre un acte de croyance, se conformer à une attitude de croyance ; jouer, c'est cet acte, cette attitude, qu'ils soient accompagnés ou non d'un contenu de croyance. C'est de l'attitude même qu'est attendu en retour un « effet » sur la réalité, une attitude positive, joyeuse, confiante permettant d'anticiper positivement l'avenir et de lui permettre alors d'advenir : «en ce sens, jouer apparaît comme un pari sur une hypothèse heureuse [...] en dépit d'une irréductible indétermination. [...] Cette incertitude attachée au jouer fonde la présence d'un enjeu au cœur de l'acte de jouer » (p. 224). Cette incertitude est responsable de la progressive valorisation des jeux à sanction interne et de la généralisation de leur orientation compétitive au fur et à mesure de la centralisation des sociétés et de l'installation de pouvoirs centralisés en recherche de stabilité et de sécurité maximale.

8 Au Jouer et au Croire, dont on aura compris que l'adoption de leur forme verbale vient de la centralité mise sur les attitudes, il faut encore ajouter, comme troisième élément indissociable des deux autres, le Métaphoriser. Les références à cette action essentielle au croire sont nombreuses. Pour ma part, je suis sensible à celle de Paul Ricœur. Il utilise ce verbe pour faire ressortir la dynamique propre de l'action métaphorique qui consiste tout autant à transgresser l'ordre catégoriel établi qu'à l'engendrer. Ricœur ne définit pas la métaphore comme étant liée à un sens figuré du mot, car le mot n'est qu'une pluralité de sens figurés. De fait, il considère inutile de chercher un sens propre originel du mot: le mot n'a pas d'essence. Utiliser une métaphore, c'est commettre un acte de transformation de l'ordre institué. La métaphore ne vaut qu'en contexte. Métaphoriser, c'est déclencher un processus dynamique capable de transformer l'appréhension de la réalité. Ainsi écrit-il, «présenter les hommes "comme agissants" et toutes choses "comme en acte", telle pourrait être la fonction ontologique du discours métaphorique ». Il est « expression vive » qui dit «l'existence vive » (Ricœur, La métaphore vive, Paris, Le Seuil, « Essais », 1975, p. 61). Le métaphoriser entretient avec le jouer et le croire la même relation ambiguë à la réalité. Elle participe, écrit Ricœur, « d'une double tension entre soumission à la réalité et invention fabuleuse ; restitution et surélévation» (idem, p. 57). Le discours métaphorique est donc, fondamentalement, interactionniste et contextuel. Il assure une transaction entre contextes par sa capacité à projeter et révéler un monde que sa performance même a transformé : la métaphore vive est nécessairement performative. Par la tension qu'elle pose entre le "n'est pas " et le " est ", elle est créatrice à sa manière d'un cadre fictionnel dont il est attendu en dehors une transformation de la perception du monde. Cette tension, conclut Ricœur, a pour conséquence une "vision stéréoscopique» de la réalité. R. Hamayon fait également le lien entre cadre fictionnel et structuration métaphorique, qu'elle articule avec la notion de marge, s'intéressant à la dimension organisationnelle, active et performative du processus métaphorique, «qui guide l'interprétation de l'expérience et façonne le comportement " (p. 305). Elle préfère cependant l'approche de George Lakoff (un linguiste) et de Mark Johnson (un philosophe). Ils inscrivent le métaphoriser dans un processus cognitif et non plus rhétorique, réservé aux mots : un geste ou un objet, sélectionné pour telle ou telle autre particularité, peuvent tout aussi bien devenir le support de la transformation relationnelle à une autre chose abstraite, invisible ou inconnue, qui ne peut être pensée directement.

9 En conclusion, dans cet ouvrage qui fait déjà référence, Roberte Hamayon a fait un triple choix : celui de s'interroger sur le processus du jouer, de considérer son unicité et 
finalement de "caractériser ce processus par l'existence d'un écart ou d'un décalage par rapport à ce qui pourrait être dans un autre ordre de réalité » (p. 320). Ce triple choix l'amène à lier l'acte de jouer, de croire et de métaphoriser et ce faisant, définissant le premier, elle participe simultanément à la construction conceptuelle des deux autres. 